\title{
A MULTIWAVELENGTH SURVEY OF INTERACTING GALAXIES
}

\author{
H. Bushouse ${ }^{1}$, S. Lamb ${ }^{2}$, K.-Y. Lo 2 , S. Lord ${ }^{3}$, and M. Werner ${ }^{3}$ \\ ${ }^{1}$ Dept. of Physics and Astronomy, Northwestern Univ., Evanston, IL \\ ${ }^{2}$ Dept. of Astronomy, University of Illinois, Urbana, IL \\ ${ }^{3}$ Space Sciences Division, NASA-Ames Res. Ctr., Moffett Field, CA
}

\section{Introduction}

Galaxy-galaxy collisions are known to produce drastic changes in morphology and, in many cases, enhance the level of star formation activity in galaxies. In order to better quantify the effects that interactions have on the star formation characteristics of galaxies we have undertaken a multiwavelength survey of a large sample of interacting disk-type galaxies. The sample is optically-selected, the inclusion of systems having been based upon the presence of unusual morphological features-such as tidal tails, plumes, rings, warped disks-suggestive of tidal interaction. The sample is composed of about 115 systems, most of which are spiralspiral pairs, with a few spiral-elliptical pairs and a few merging systems (see Bushouse 1986 for more details of the sample selection). This sample has now been studied in the optical, infrared, and radio regimes, including optical spectra and $\mathrm{H} \alpha$ images, near-infrared photometry and imaging, far-infrared photometry, $\mathrm{H} \mathrm{I} 21 \mathrm{~cm}$ emission-line measurements, VLA $20 \mathrm{~cm}$ maps, and $\mathrm{CO}$ emission-line measurements. This paper presents an overview and comparison of the results of the optical, infrared and CO surveys. With these data we can compare the far-infrared and $\mathrm{CO}$ properties of the galaxies with the classic optical and radio indicators of star formation activity and thereby determine what, if any, relationships exist between star formation activity and the far-infrared and $\mathrm{CO}$ properties of the galaxies.

\section{Optical Properties}

Global star-formation rates (SFRs) derived from $\mathrm{H} \alpha$ emission-line luminosities span a wide range with a large fraction of the interacting galaxies having SFRs indistinguishable from that of isolated spirals (Bushouse 1987). On average, however, the global SFRs of the interacting galaxies are a factor of 2.5 higher than that of isolated spiral galaxies of similar luminosity. The level of enhanced activity does not depend strongly on either galaxy size or the proximity of companions, although high levels of star formation activity are seen more often in close pairs. All possible combinations of activity, i.e. pairs in which both galaxies are active or inactive and pairs in which only one galaxy is active, appear to occur in roughly equal numbers.

Quantitative analysis of the spatial distribution of $\mathrm{H} \alpha$ emission in the interacting galaxies indicates that the majority of interaction-induced star formation activity occurs preferentially in the near-nuclear regions. Modest enhancements in the level of disk star formation are also seen to occur in a few systems. Given the fact that the radial distribution of star-forming regions in isolated spirals approximately follows the integrated light of the stellar disk (Hodge and Kennicutt 1983), we conclude that interaction-induced star formation usually does not 
follow the same pattern as preinteraction star-formation activity.

Emission-line ratios of most nuclear region spectra are consistent with photoionization by $\mathrm{H}$ II regions, with only a few showing LINER and Seyfert characteristics. Thus it appears that in the vast majority of systems in this sample interactions are triggering "normal" modes of star formation activity, while the creation or activation of nuclear "monsters" occurs infrequently.

\section{Far-Infrared Properties}

Far-infrared (42-122 $\mu \mathrm{m})$ luminosities derived from IRAS data for the interacting systems again cover a wide range, showing considerable overlap with the luminosities of isolated spirals (Bushouse, Lamb and Werner 1988). However, the median far-infrared luminosity of the interacting galaxies is about a factor of four greater than that of a sample of isolated spiral galaxies. Some of this increase is most certainly due to the fact that the sample of interacting galaxies is somewhat biased towards intrinsically more luminous galaxies, therefore the ratio of infrared-to-optical luminosity is a more appropriate property for comparison. We find that the median $L_{\mathrm{IR}} / L_{B}$ value for the interacting galaxies is about a factor of two greater than that of isolated spirals. Thus, by this measure, interactions are producing an approximate doubling of infrared luminosity. Assuming that this increase is tied to increased heating of interstellar dust due to enhanced star formation, this result is entirely consistent with the factor of two or so increase in optically-derived star formation rates which was found previously.

The median $12 \mu \mathrm{m} / 25 \mu \mathrm{m}$ and $60 \mu \mathrm{m} / 100 \mu \mathrm{m}$ colors of the interacting galaxies are also different from those of isolated spirals, with the interacting galaxies having larger $60 \mu \mathrm{m} / 100 \mu \mathrm{m}$ and smaller $12 \mu \mathrm{m} / 25 \mu \mathrm{m}$ flux ratios. The higher $60 \mu \mathrm{m} / 100 \mu \mathrm{m}$ flux ratios, which imply higher dust temperatures, are consistent with the assumption that the increase in far-infrared emission is due to an increase in the population of young stars.

Two curious phenomena are revealed when comparing the relationship between $L_{\mathrm{IR}} / L_{B}$ and $L_{B}$ for the interacting and isolated galaxy samples. First, there are no isolated galaxies, of any optical luminosity, with $L_{\mathrm{IR}} / L_{B}$ much greater than 10 , whereas fully one-third of the interacting systems have $L_{\mathrm{IR}} / L_{B}$ ratios above this level and several have $L_{\mathrm{IR}} / L_{B}>30$ (see figure 6 in Bushouse, Lamb and Werner 1988). Therefore it is likely that galaxy-galaxy interactions are a necessary requirement for significant enhancements in infrared luminosity. Second, there appears to be a cutoff at $L_{B} \sim 10^{9} L_{\odot}$, below which no systems-neither interacting nor isolated-show a significant enhancement in infrared luminosity. This result is consistent with Smith et al. (1987) who find that galaxies of low blue luminosity are not strong infrared emitters.

We have searched for correlations between classic optical indicators of star-formation activity and far-infrared properties in order to determine to what extent the far-IR emission is driven by star formation. We have found that on an individual galaxy-by-galaxy basis there is only a modest correlation between the star-formation rate per unit area of a galaxy (as derived from $\mathrm{H} \alpha$ emission-line luminosities) and the far-infrared "excess" $\left(L_{\mathrm{IR}} / L_{B}\right)$ and infrared colors. There is a much stronger correlation, however, between star-formation activity and both $L_{\mathrm{IR}} / L_{B}$ and far-infrared colors when the sample of galaxies is broken into groups based on their optical spectral characteristics. Those galaxies having spectra characteristic of an 
old "dormant" stellar population have an average $L_{\mathrm{IR}} / L_{B} \sim 2.5$ and very low $60 \mu \mathrm{m} / 100 \mu \mathrm{m}$ and $25 \mu \mathrm{m} / 12 \mu \mathrm{m}$ flux ratios, while galaxies showing strong $\mathrm{H}$ II region-type emission features have an average $L_{\mathrm{IR}} / L_{B} \sim 14$ and $60 \mu \mathrm{m} / 100 \mu \mathrm{m}$ and $25 \mu \mathrm{m} / 12 \mu \mathrm{m}$ flux ratios a factor of $2-3$ higher than galaxies with low SFRs. Systems that appear to be in the process of merging into a single body have the highest values of all, with $L_{\mathrm{IR}} / L_{B} \sim 55$ and colors a factor of $3-4$ higher than low SFR systems.

While the majority of interacting pairs are too closely spaced to be resolved by IRAS, detailed analysis of the IRAS data has allowed us to measure the levels of $25 \mu \mathrm{m}$ and $60 \mu \mathrm{m}$ emission from the individual galaxies in approximately 20 pairs. Contrary to the results of Telesco, Wolstencroft, and Done (1988), we find that in most spiral-spiral pairs both galaxies are far-infrared emitters. It is usually the case, however, that one of the galaxies is brighter at $60 \mu \mathrm{m}$ than its companion by a factor of 2-4, although in a few pairs both galaxies are equally bright.

\section{Molecular Gas Properties}

CO $(J=1-0)$ emission-line observations have been obtained for 24 of the interacting systems with the NRAO 12-m telescope (Bushouse et al. 1990). CO observations for another 11 systems in the present sample are available in the literature. In agreement with previous studies, we find a strong correlation between far-infrared luminosity and molecular gas content for the interacting systems that have been observed. We also find strong correlations between the FIR luminosity-to-molecular gas ratio and both the $60 \mu \mathrm{m} / 100 \mu \mathrm{m}$ flux ratio and $L_{\mathrm{IR}} / L_{B}$. The molecular gas contents of the interacting galaxies span a large range but are, on average, a factor of 3-4 higher than that of isolated spiral galaxies. These systems also have an average $L_{\mathrm{IR}} / M\left(\mathrm{H}_{2}\right)$ ratio about a factor of 2 higher than isolated spirals, which others have interpreted as a higher SFR per unit gas mass for interacting galaxies (e.g. Young et al. 1986; Solomon and Sage 1988). Systems suspected to be in the process of merging-e.g. Arp 193, Arp 243, and $\operatorname{Arp} 299$ - have the largest $L_{\mathrm{IR}} / M\left(\mathrm{H}_{2}\right)$ values in the sample. The molecular-toatomic gas ratio, $M\left(\mathrm{H}_{2}\right) / M(\mathrm{H} \mathrm{I})$, has a relatively constant value of $\sim 1.5$ among the systems that have been observed and does not depend strongly on $L_{\mathrm{IR}} / L_{B}$, which has been found to correlate with star-formation activity (§III). Only those systems with very low $L_{\mathrm{IR}} / L_{B}$ also have unusually low $M\left(\mathrm{H}_{2}\right) / M(\mathrm{H} \mathrm{I})$ ratios $\lesssim 0.4$. Therefore systems with higher SFRs are not necessarily overabundant in terms of molecular gas relative to atomic gas.

A comparison of optically-determined SFRs with the molecular gas content of the interacting galaxies shows that there is a modest correlation between the ratio of SFRs for the two galaxies in an individual pair and the ratio of molecular gas content for that pair of galaxies. In other words, it appears that the relative molecular gas content of the two galaxies in a pair is at least partially responsible for the relative level of interaction-induced star-formation activity. There are exceptions to this, however, as there are some pairs composed of two galaxies with nearly equal molecular gas content and yet one galaxy has a SFR as much as 9 times higher than its companion. Therefore, orbital and other internal properties of the galaxies must also play a role in determining the level of interaction-induced star-formation activity. 


\section{Unusual Interacting Systems}

In spite of the correlations between various observable properties as discussed in the previous sections there are, of course, some systems that do not fit the general trends. As is often the case, these exceptions can reveal many interesting properties. First, while many of the interacting galaxies have very high SFRs, there are also many systems that have very low optically-determined SFRs and low far-infrared luminosities. Furthermore, many of these low SFR galaxies occur in pairs with a companion that has a very high SFR. These low SFR galaxies have optical luminosities that are typical for normal isolated spirals and also have normal atomic H I gas contents, while their molecular gas contents span a large range. Therefore these are not intrinsically small or "anemic" galaxies and so the low levels of star formation activity are not simply due to a lack of fuel. Orbital and other internal properties must again play a role here.

Second, while we found that galaxies with optical indications of high SFRs usually have significantly higher than average $L_{\mathrm{IR}} / L_{B}$ and "warm" far-infrared colors, there are several systems with strong $H$ II region-type spectra that have very low far-infrared luminosities and cool colors. Further analysis has shown that these systems have low optical luminosities $\left(L_{B}\right.$ $\left.\sim 5 \times 10^{8} L_{\odot}\right)$, but relatively normal atomic gas content $\left(M(\mathrm{H} \mathrm{I}) \sim 4 \times 10^{9} M_{\odot}\right)$. Optical emission-line ratios indicate that these galaxies are metal-poor, having $\mathrm{O} / \mathrm{H}$ ratios 3-4 times lower than the high SFR systems that have high far-infrared luminosities. CO observations of these systems have yielded null detections, indicating very low molecular gas contents of $M\left(\mathrm{H}_{2}\right)<10^{9} M_{\odot}$. Therefore, the lack of far-infrared emission from these galaxies may be due to the absence of a sufficiently massive ISM necessary to convert UV/optical radiation into the far-infrared. Futhermore, if these galaxies maintain their current SFRs, they will exhaust their molecular gas supplies in a few $\times 10^{8}$ years.

\section{Near-Infrared Imaging}

We have obtained $J(1.25 \mu \mathrm{m})$ and $K(2.2 \mu \mathrm{m})$ images of about 20 of the interacting galaxies using the Kitt Peak National Observatory $58 \times 62$ element infrared camera (Bushouse and Werner 1990). In most systems the large-scale infrared morphology is similar to that seen in optical continuum images. However, in many systems there are small-scale features that are strikingly different from their optical counterparts, which we believe to be due mainly to the obscuring effect of dust at optical wavelengths. In some galaxies the infrared images have revealed the locations of previously invisible nuclei. In NGC 520, for example, the infrared images show the primary nucleus to be located at the very center of the massive dust lane that dominates optical images of the system. They also suggest that the fainter concentration to the northwest may be the nuclear remnant of a second galaxy. In general there is little correlation between the near-infrared and $\mathrm{H} \alpha$ morphologies of the galaxies. Only the very brightest H II complexes stand out from the background stellar population in the infrared images.

An analysis of the spatial distribution of infrared colors has shown that virtually all of the galaxies become redder towards their nuclei. On average, the nuclei are 0.4 and 0.9 mag redder in $J-K$ and $R-K$, respectively, than the disk regions. The majority of this effect appears to be due to an increase in the amount of dust in the nuclei of the galaxies. The ratio of color changes in $R-K$ and $J-K$ is, however, somewhat less than the value for reddening 
by dust alone, suggesting that metallicity and population gradients are also present. The "excess" reddening in $J-K$ relative to $R-K$ might also arise if the dust is well mixed with the stars, in which case the infrared colors would have a larger contribution from stars with large amounts of extinction. It appears that little, if any, of the red nuclear colors is due to thermal emission from dust since of the two galaxies in the sample that are experiencing the largest nuclear starbursts-NGC 1614 and NGC 7714-neither show unusually red nuclear colors relative to lower SFR systems.

\section{References}

Bushouse, H. A. 1986, Ph.D. thesis, University of Illinois. 1987, Ap.J., 320, 49.

Bushouse, H. A. and Werner, M. W. 1990, Ap.J., in press.

Bushouse, H. A., Lamb, S. A. and Werner, M. W. 1988, Ap.J., 335, 74.

Bushouse, H. A., Lamb, S. A., Lo, K.-Y., Lord, S. D. and Werner, M. W. 1990, in preparation. Hodge, P. W. and Kennicutt, R. C. 1983, Ap.J., 267, 563.

Smith, B. J., Kleinmann, S. G., Huchra, J. P. and Low, F. J. 1987, Ap.J., 318, 161.

Solomon, P. M. and Sage, L. J. 1988, Ap.J., 334, 613.

Telesco, C. M, Wolstencroft, R. D., and Done, C. 1988, Ap.J., 329, 174.

Young, J. S. et al. 1986, Ap.J.(Letters), 311, L17. 


\section{DISCUSSION}

Osterbrock: I believe the relatively small number of AGNs or seyfert galaxies which you mentioned can be understood as resulting from the fact that your sample is selected as strongly interacting, violently disturbed systems. Dahari and others have shown observationally that mild interactions tend to enhance seyfert activity, but violent interactions do not. Presumably the explanation is connected with the situations in which gas can be delivered near the nucleus with nearly zero angular momentum.

Bushouse: Yes, this result may be consistent with the study by Dahari, although it's interesting to note that the few liner's in my sample seem to occur preferentially in the on-going merger systems which are obviously the most strongly interacting systems. I think the sample of ultraluminous IRAS galaxies studied by Sanders et al. also shows evidence for larger than normal contributions from AGN-type activity.

Zasov: Can you compare briefly the behavior of late- and earlytype interacting galaxies? For example, is there any noticable difference between fractions of galaxies which have experienced enhanced SF among different morphological types?

Bushouse: Unfortunately the disturbed morphologies of many of these galaxies do not allow us to accurately classify them by Hubble sub-types. However, I believe that in their sample, Keel and Kennicutt did determine Hubble sub-types and essentially found no correlation between star formation activity and morphological type across the spiral sequence.

Rennicutt: (Comment in reply to a question from zasov to Bushouse - dependence of star formation on morphological type.) In our study the distributions of $\mathrm{H}$-alpha emission are nearly independent of Hubble type. However there may be some differences in the spatial distributions of the emission. In those early-type spirals (sa-sb) which have high levels of emission the activity is often confined to the nuclear regions. 